\title{
COMPARAÇÃO ENTRE DOIS MÉTODOS PARA A DETERMINAÇÃO DO VOLUME DE ESCOAMENTO SUPERFICIAL ${ }^{(1)}$
}

\author{
F. F.PRUSKI (2), N.P. GRIEBELER ${ }^{(3)} \&$ D. D. SILVA(2)
}

\begin{abstract}
RESUMO
A determinação do volume máxi mo de escoamento superficial é fundamental para o dimensionamento de diversos tipos de obras hidráulicas, constituindo o parâmetro de maior importância no projeto de sistemas de terraceamento em nível. A determinação incorreta do volume escoado superficialmente gera imprecisões no dimensionamento destes sistemas que, quando superdimensionados, apresentam elevado custo de instalação. Tendo em vista esses aspectos, o presente trabalho teve por objetivo comparar os valores de volume máximo de escoamento superficial, obtidos por meio do método empírico do número da curva, desenvolvido pelo Serviço de Conservação de Solos dos Estados Unidos, com aqueles obtidos por meio do método desenvolvido por Pruski e colaboradores, baseado em princípios consagrados na hidrologia. 0 desempenho dos dois métodos foi analisado com base nas condições pluviométricas correspondentes a Belo Horizonte e Uberlândia, para período de retorno de 10 anos. Evidenciaram-se grandes diferenças entre os valores de volume de escoamento superficial obtidos pelos dois métodos, as quais se mostraram mais acentuadas para maiores velocidades de infiltração de água no solo, tendo o método proposto por Pruski e colaboradores apresentado comportamento mais condizente com a realidade.
\end{abstract}

Termos de indexação: conservação de solos, terraceamento, modelos hidrológicos.

(1) Recebido para publicação em outubro de 1999 e aprovado em janeiro de 2001.

(2) Professor do Departamento de Engenharia Agrícola da Universidade Federal de Viçosa - UFV. CEP $36571-000$ Viçosa (MG). Bolsista do CNPq. E-mail: ffpruski@mail.ufv.br; david@mail.ufv.br

(3) Doutorando em Engenheira Agrícola do Departamento de Engenharia Agrícola, UFV. Bolsista da CAPES. 


\title{
SUMMARY: COMPARISON BETWEEN TWO METHODS TOESTIMATE THE SURFACE RUNOFF VOLUME
}

\begin{abstract}
Theestimativeof themaxi mum surfacerunoff vol umeis fundamental to design several types of hydraulic structures as, for example, level terracing systems. Theincorrect estimative of the surfacerunoff vol umecauses a wrong design of thesesystems and when overestimated present high installation cost. Considering theseaspects, thepresent study ai med to compare thevalues of thesurfaceflow maximum vol umeobtained by theempiric method of thecurve number and developed by the USA Soil Conservation Service, with those obtained by application of themethodol ogy devel oped by Pruski and collaborators, based on hydrol ogyconsecrated principles. Theperformance of thetwo methods was analyzed by considering a 10-year return period and therain pluvi ometric conditions corresponding to Bel o H orizonte and U berlândia cities. Great differences were evidenced among the values of the surface flow vol ume obtained by both methods, which were shown more accentuated for soil water intakerates, and the method proposed by Pruski and his collaborators presented behavior according to reality.
\end{abstract}

Index terms: soil conservation, terracing, hydrol ogic models.

\section{NTRODUÇÃO}

A principal causa da degradação das terras agrícolas é a erosão do solo, a qual consiste nos processos de desprendimento e arraste das partículas de solo causados pela ação da água e do vento. No Brasil, a erosão hídrica é a mais importante, sendo responsável pela maior partedas perdas de sol o. O escoamento superficial transporta partículas de solo em suspensão, nutrientes, matéria orgânica, sementes e defensivos agrícolas, prejudicando a produção agropecuária e poluindo os recursos hídricos (Bertoni \& Lombardi N eto, 1990).

Para dimensionar drenos, obras de proteção contra cheias e erosão hídrica, torna-se necessário o conhecimento de vári ios fatores, dos quais o principal é o escoamento superficial. Lombardi Neto et al. (1994) afirmam que, para o dimensionamento de terraços com gradiente, a taxa de escoamento superficial é o parâmetro de maior importância, enquanto, para o projeto de terraços em nível, o volume de escoamento é o fator mais importante.

Existem muitos procedimentos para obter o escoamento superficial, os quais, na maioria das vezes, são desenvolvidos para estimar as vazões máximas obtidas em bacias hidrográficas. Um método simples para a determinação do volume de escoamento superficial é o método proposto pelo Serviço de Conservação de Solos do Departamento de Agricultura dos Estados Unidos (SCS-USDA). Este método é apresentado na literatura como método do número da curva, exigindo o conhecimento prévio da preci pitação e do complexo hidrológi co solo-vegetação (Rojas, 1984).
O método do número da curva foi desenvolvido com base em estudos empíricos realizados em diversas regiões dos Estados Unidos, considerando diferentes condições e tipos de cobertura do solo (Rallison, 1980). Este método, conforme Wells et al . (1986), é utilizado para estimar o volume de escoamento superficial em pequenas bacias hidrográficas em que sejam disponíveis informações relativas às condições do solo e da vegetação e registros diários de precipitação. Apresenta, portanto, a vantagem de requerer informações de fácil obtenção para ser utilizado.

Estudo realizado pel o "Water Resources Council”, citado por Bonta \& Rao (1992), relata a dificuldade para a aplicação dos procedimentos disponíveis para a estimativa do escoamento superficial em virtude da imprecisão de alguns métodos usualmente utilizados para esta finalidade, bem como da grande variabilidade na estimativa que pode ser obtida por diferentes profissionais que usem o mesmo procedimento.

Pruski et al. (1997) desenvolveram um método com base em fundamentos da engenharia para determinar a lâmina máxima de escoamento superficial. Utilizaram, para tanto, uma equação que descreve o balanço de água na superfície do solo. Martins J r. (1997), comparando resultados calculados por este método com os obtidos experimentalmente, observou que este permite obter boas estimativas da lâmina máxima de escoamento superficial.

Tendo em vista os al tos custos de instalação de obras hidráulicas e de combate à erosão e a grande dependência que seu dimensionamento tem do 
volume de escoamento superficial, o presente trabal ho foi desenvolvido com o objetivo de comparar os valores da lâmina máxima de escoamento superficial, obtidos pelo método do número da curva, com aqueles calculados pelo método proposto por Pruski et al. (1997).

\section{MATE RIAL E MÉTODOS}

\section{Método do número da curva (CN)}

Para estimar o escoamento superficial, o método do número da curva (CN) utiliza informações relativas à chuva e ao complexo hidrológico solovegetação definido pelo SCS-USDA, que considera o tipo, o uso, a umidade inicial e a condição hidrológica do solo. Os componentes associados a este método estão representados na figura 1.

O método do número da curva foi desenvolvido a partir da análise degrande número de chuvas, sendo o escoamento superficial expresso pela equação

$$
\mathrm{ES}=\frac{\left(\mathrm{PT}-\mathrm{I}_{\mathrm{a}}\right)^{2}}{\mathrm{PT}+0,8 \mathrm{~S}}
$$

em que

ES = escoamento superficial, $\mathrm{mm}$;

PT = preci pitação total, $\mathrm{mm}$;

$\mathrm{I}_{\mathrm{a}}=$ abstrações iniciais, $\mathrm{mm}, \mathrm{e}$

$\mathrm{S}=$ capacidade máxima de infiltração, $\mathrm{mm}$.

Visando à simplificação do emprego do método do número da curva, a precipitação recomendada para uso no método éaquel a correspondente ao total precipitado para determinado tempo de retorno e duração de preci pitação requerido (normal mente 6, 12 ou $24 \mathrm{~h}$ ), sendo determinada a partir de dados pluviométricos.

A capaci dade máxima de infiltração é obtida pela equação

$$
\mathrm{S}=\left[\frac{25400}{\mathrm{CN}}\right]-254
$$

em que $\mathrm{CN}$ é o número da curva, que define o complexo hidrológico solo-vegetação, e os valores numéricos são coeficientes para ajuste de unidades.

Quando do desenvolvimento do método do número da curva, foi constatado que a precipitação antecedente ao escoamento superficial, designada abstrações iniciais $\left(I_{a}\right)$, representa aproximadamente $20 \%$ da capacidade máxima de infiltração da água no solo, sendo descrita pela equação

$$
\mathrm{I}_{\mathrm{a}}=0,2 \mathrm{~S}
$$

Para determinar o volume de escoamento superficial pel o método do número da curva, utilizouse, neste trabal ho, a condição de sol o sem cultivo ou descoberto e a precipitação acumulada, anterior à ocorrência da chuva considerada para realização de projetos superior a $52,5 \mathrm{~mm}$, indicando solo com umidade próxima à de saturação. $\mathrm{O}$ método $\mathrm{CN}$ permite determinar o número da curva para quatro grupos de solos (A, B, C e D), divididos conforme a capacidade de produção de escoamento superficial. Estes grupos de solos foram definidos pelo SCSUSDA. O risco de ocorrência de escoamento superficial aumenta no sentido de A para D, enquanto a capacidade de infiltração se reduz. Para as condições consideradas, os valores de $\mathrm{CN}$ são iguais a: 89, para o grupo A; 94, para o grupo B; 97 , para o grupo C, e 98, para o grupo D.

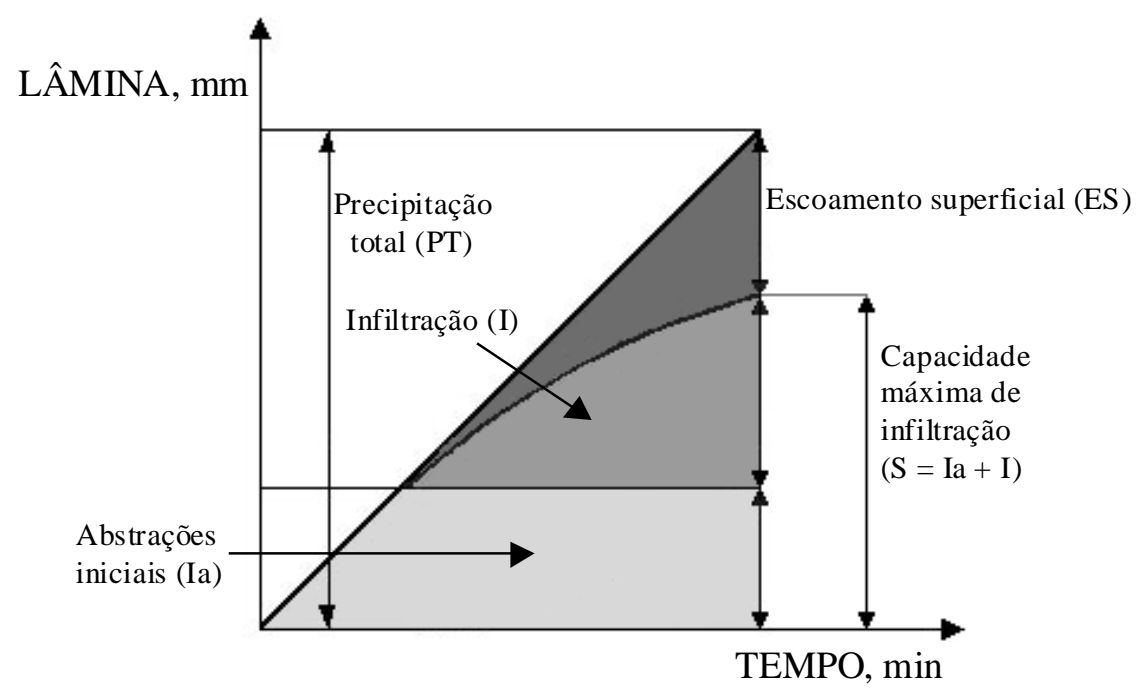

Figura 1. Componentes associados ao método do número da curva, sugerido pelo serviço de conservação de solo do Departamento de Agricultura dos Estados Unidos (SCS-USDA). 
Diversos pesquisadores têm proposto como critério para diferenciação dos grupos de solos o estabel ecimento de limites de taxas de infiltração. Estes limites, entretanto, apresentam grandes variações, quando considerados diferentes autores. Hawkins (1980) listou al guns dos limites propostos, os quais são apresentados no quadro 1. Pruski et al. (1997) utilizaram para a classificação do solo nos diferentes grupos definidos pelo SCS-USDA os seguintes valores de taxa de infiltração básica da água no solo: grupo A - valores maiores que $190 \mathrm{~mm} \mathrm{~h}^{-1}$, grupo B - valores entre $40 \mathrm{~mm} \mathrm{~h}^{-1} \mathrm{e}$ $190 \mathrm{~mm} \mathrm{~h}^{-1}$, grupo C - valores entre $3 \mathrm{~mm} \mathrm{~h}^{-1} \mathrm{e}$ $40 \mathrm{~mm} \mathrm{~h}^{-1}$, e grupo $\mathrm{D}$ - valores menores que $3 \mathrm{~mm} \mathrm{~h}^{-1}$.

\section{Método desenvolvido por Pruski et al. (1997)}

A determinação da lâmina máxima deescoamento superficial por este método é feita pela equação

$$
\mathrm{ES}=\mathrm{PT}-\mathrm{I}_{\mathrm{a}}-\mathrm{I}
$$

em quel =infiltração acumulada, $\mathrm{mm}$.

No cál culo da precipitação total, utiliza-se a equação

$$
\mathrm{PT}=\frac{\mathrm{i}_{\mathrm{m}} \mathrm{t}}{60}
$$

em que $i_{m}=$ intensidade máxima média de preci pitação, $\mathrm{mm} \mathrm{h}^{-1}$, et =duração da precipitação, min.

A intensidade máxima média de precipitação é obtida pela equação

$$
i_{m}=\frac{K T^{a}}{(t+b)^{c}}
$$

em que

$\mathrm{K}, \mathrm{a}, \mathrm{b}, \mathrm{c}=$ parâmetros de ajuste da equação relativos à estação pluviográfica, e T = período de retorno, anos.
Substituindoa equação 6 na equação 5 e derivando em relação ao tempo, obtém-se a intensidade de preci pitação instantânea $\left(\mathrm{i}_{\mathrm{i}}\right), \mathrm{em} \mathrm{mm} \mathrm{h}^{-1}$, no instante t. Portanto

$$
\mathrm{i}_{\mathrm{i}}=\mathrm{i}_{\mathrm{m}}\left(1-\frac{\mathrm{ct}}{\mathrm{t}+\mathrm{b}}\right)
$$

Pela análise das equações 6 e 7, observa-se que tanto $i_{m}$ como $i_{i}$ dimi nuem com o aumento det, sendo oES máximo aquel e correspondente ao instante em que $i_{i}$ se iguala à vel ocidade de infiltração básica da água no solo (VIB), em mm h-1. Para esta condição, tem-se

$$
\mathrm{i}_{\mathrm{m}}\left(1-\frac{\mathrm{ct}}{\mathrm{t}+\mathrm{b}}\right)-\mathrm{VIB}=0
$$

A partir desta equação, calculou-se o valor de t por meio do método de convergência de NewtonRaphson.

A precipitação total correspondente a esta duração foi cal culada pela equação 5 e as abstrações iniciais foram calculadas pela equação 3.

A duração das abstrações iniciais $\left(t_{l a}\right)$, em min, foi calculada pela equação

$$
\int_{0}^{\mathrm{t}} \mathrm{Ia} \mathrm{i}_{\mathrm{i}} \mathrm{dt}=\mathrm{I}_{\mathrm{a}}
$$

Para a sol ução da equação 9 também foi utilizado o método de Newton-Raphson.

Obteve-se a duração da infiltração ( inf $_{\text {inf }}$, em min, subtraindo da duração da preci pitação para a qual o escoamento superficial é máximo (t) o valor da duração das abstrações iniciais, ou seja, $t_{\text {inf }}=t-t_{1 a}$. A infiltração acumulada (I), em mm, foi calculada pela equação

$$
\mathrm{I}=\frac{\mathrm{VIB} \mathrm{t}_{\mathrm{inf}}}{60}
$$

\section{Quadro 1. Taxas de infiltração de água no solo propostas por diferentes pesquisadores para a classificação do solo, segundo os grupos definidos pelo SCS-USDA para utilização no método do número da curva}

\begin{tabular}{lcccc}
\hline & \multicolumn{4}{c}{ Taxa de infiltração para os diferentes grupos de solo (mm h-1) } \\
\cline { 2 - 4 } \multicolumn{1}{c}{ Autor } & D & C & B & A \\
\hline Musgrave, 1955 & $<1,3$ & $1,3-3,8$ & $3,8-7,6$ & $>7,6$ \\
SCS, 1972 & $<5,1$ & $5,1-20,3$ & $20,3-127,0$ & $>127,0$ \\
Leven \& Stender, 1967 & $<20,3$ & $20,3-63,5$ & $63,5-127,0$ & $>127,0$ \\
USFS, sem data & $<12,7$ & $12,7-31,8$ & $31,8-76,2$ & $>76,2$ \\
Miller et al., 1973 & $<2,0$ & $2,0-3,8$ & $3,8-7,6$ & $>7,6$ \\
Estgate, 1977 & $<4,6$ & $4,6-9,9$ & $9,9-30,0$ & $>30,0$ \\
Musgrave, 1973 & $<5,6$ & $5,6-11,9$ & $11,9-25,4$ & $>25,4$ \\
\hline
\end{tabular}

Fonte: Hawkins (1980). 
Os componentes associados ao método desenvolvido por Pruski et al. (1997) estão representados na figura $2 a$. Na figura $2 b$, pode-se evidenciar a sensibilidade do método representada pela redução do escoamento superficial ao aumento das abstrações iniciais que pode ser obtido com o aumento da cobertura vegetal ou da capacidade dearmazenamento de água na superfície do terreno. Na figura 2c, pode-se observar a redução do escoamento superficial com o aumento da velocidade de infiltração básica da água no solo.

\section{Comparação entre os métodos}

Visando comparar os dois métodos, utilizaramse, nesteestudo, as informações rel ativas às equações deintensidade, duração efreqüência da preci pitação, obtidas por Pinto et al. (1996) para as localidades de Belo Horizonte (equação 11) eU berlândia (equação 12).

$$
\begin{aligned}
& i_{m}=\frac{1175,295 T^{0,255}}{(t+13,381)^{0,806}} \\
& i_{m}=\frac{1167,284 T^{0,233}}{(t+17,245)^{0,747}}
\end{aligned}
$$

Tendo em vista o fato de serem as equações de intensidade, duração e freqüência da precipitação conhecidas para as local idades estudadas, e visando mel hor comparar os métodos, utilizaram-setambém as equações 4 e 5 para estimar a preci pitação total para o método CN. Para tanto, usaram-seas durações de precipitação de 6, 12 e 24 h.
Foi consi derado um período de retorno de 10 anos, muito utilizado no dimensionamento de sistemas de conservação de solos e drenagem de superfície. As faixas (intervalos) de VIB consideradas para a classificação do solo nos grupos propostos para o método CN foram as sugeridas por Pruski et al. (1997), fazendo-se, posteriormente, uma comparação com os resultados obtidos a partir do uso dos critérios propostos pelo SCS, 1972, (Quadro 2).

A comparação entre os métodos foi feita com base nos valores da lâmina máxima de escoamento superficial produzida, a qual representa o volume máximo deescoamentosuperficial por unidadedeárea.

\section{RESULTADOS E DISCUSSÃO}

No quadro 2, são apresentados, para a cidade de Belo Horizonte, os valores da lâmina máxima de escoamento superficial, obtidos pelos dois métodos estudados para a estimativa do escoamento superficial máximo, considerando os dois critérios utilizados para a classificação do solo nos grupos definidos pelo SCS-USDA. Nas figuras 3 e 4, encontram-se as variações dos valores de lâmina máxima de escoamento superficial em função da velocidade de infiltração básica deágua nosolo(VIB), utilizando os dois métodos estudados para a estimativa do escoamento superficial máximo, para Bel o H orizonte e U berlândia, respectivamente.

Quadro 2. Valores da lâmina máxima de escoamento superficial, em mm, para Belo Horizonte, obtidos pelo método proposto por Pruski et al. (1997) e pelo método do número da curva (CN), consi derando o critério proposto por Pruski et al. (1997) e SCS para a classificação dos solos conforme a velocidade

\begin{tabular}{|c|c|c|c|c|c|c|c|c|}
\hline \multirow{4}{*}{ VIB } & \multicolumn{8}{|c|}{ Critério utilizado para a classificação do solo nos grupos propostos pelo SCS-USDA } \\
\hline & \multicolumn{4}{|c|}{ Pruski et al. (1997) } & \multicolumn{4}{|c|}{ SCS } \\
\hline & \multirow{2}{*}{$\begin{array}{c}\text { Pruski et al. } \\
\text { (1997) }\end{array}$} & \multicolumn{3}{|c|}{$\mathbf{C N}$} & \multirow{2}{*}{$\begin{array}{c}\text { Pruski et al. } \\
\text { (1997) }\end{array}$} & \multicolumn{3}{|c|}{$\mathbf{C N}$} \\
\hline & & $6 \mathrm{~h}$ & $12 \mathrm{~h}$ & $24 h$ & & $6 h$ & $12 \mathrm{~h}$ & $24 h$ \\
\hline \multicolumn{9}{|c|}{$\mathrm{mm} \mathrm{h}^{-1}$} \\
\hline 1 & 119,1 & 101,2 & 118,4 & 137,3 & 119,1 & 101,2 & 118,4 & 137,3 \\
\hline 2 & 99,7 & 101,2 & 118,4 & 137,3 & 99,7 & 101,2 & 118,4 & 137,3 \\
\hline 3 & 89,4 & 101,2 & 118,4 & 137,3 & 89,4 & 101,2 & 118,4 & 137,3 \\
\hline 5 & 76,7 & 98,3 & 115,5 & 134,4 & 77,1 & 101,2 & 118,4 & 137,3 \\
\hline 10 & 60,9 & 98,3 & 115,5 & 134,4 & 60,9 & 98,3 & 115,5 & 134,4 \\
\hline 20 & 45,4 & 98,3 & 115,5 & 134,4 & 45,4 & 98,3 & 115,5 & 134,4 \\
\hline 35 & 32,9 & 98,3 & 115,5 & 134,4 & 31,9 & 89,9 & 106,9 & 125,6 \\
\hline 50 & 24,1 & 89,9 & 106,9 & 125,6 & 24,1 & 89,9 & 106,9 & 125,6 \\
\hline 65 & 18,5 & 89,9 & 106,9 & 125,6 & 18,5 & 89,9 & 106,9 & 125,6 \\
\hline 85 & 13,1 & 89,9 & 106,9 & 125,6 & 13,1 & 89,9 & 106,9 & 125,6 \\
\hline 100 & 10,0 & 89,9 & 106,9 & 125,6 & 10,0 & 89,9 & 106,9 & 125,6 \\
\hline 125 & 6,3 & 89,9 & 106,9 & 125,6 & 6,3 & 89,9 & 106,9 & 125,6 \\
\hline 150 & 3,7 & 89,9 & 106,9 & 125,6 & 3,0 & 76,9 & 93,3 & 111,5 \\
\hline 175 & 1,9 & 89,9 & 106,9 & 125,6 & 1,4 & 76,9 & 93,3 & 111,5 \\
\hline 210 & 0,3 & 77,0 & 93,3 & 111,5 & 0,3 & 76,9 & 93,3 & 111,5 \\
\hline
\end{tabular}
de infiltração básica da água no solo (VIB) nos diferentes grupos propostos pelo SCS-USDA 

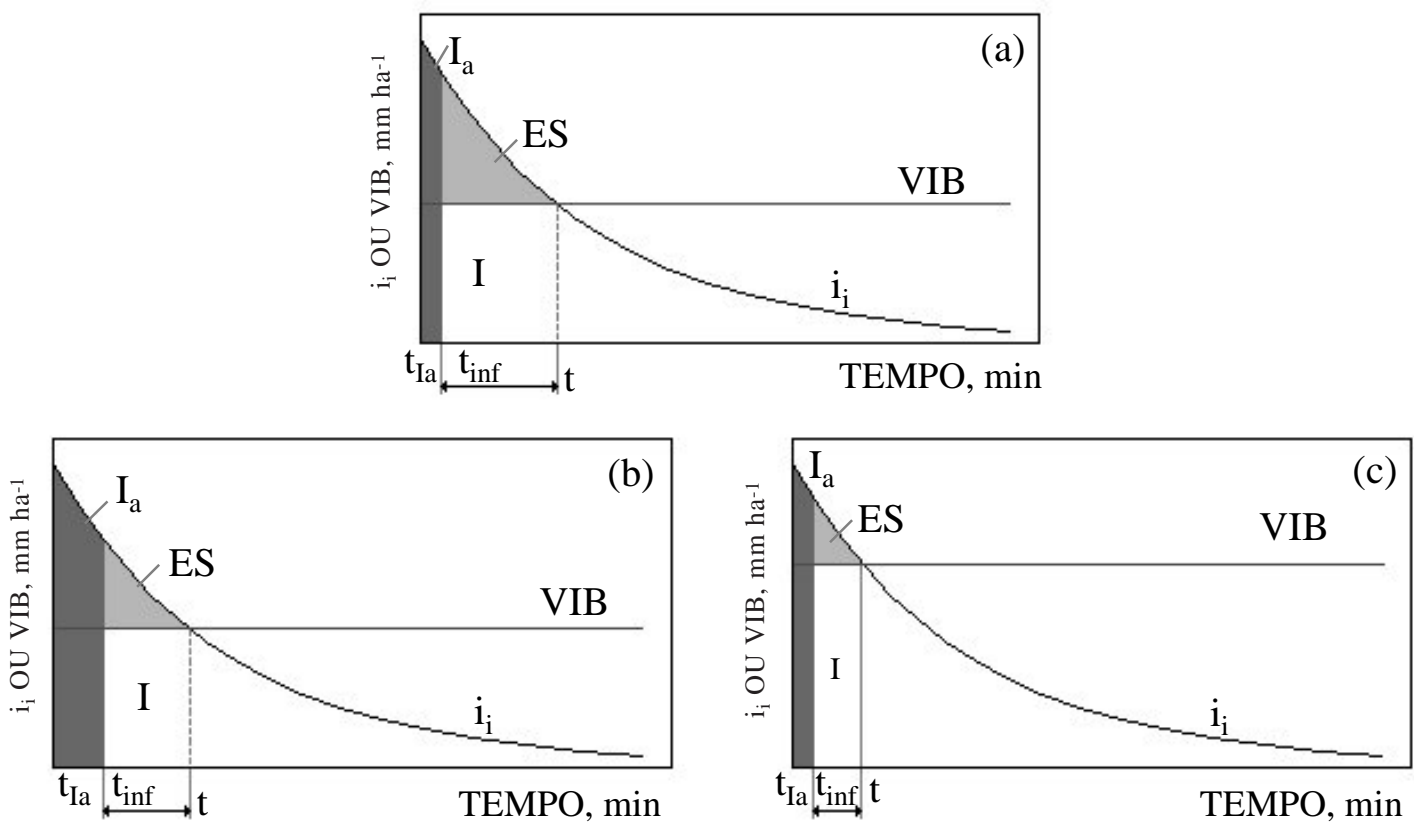

Figura 2. Componentes associados ao método proposto por Pruski et al. (1997) (a); representação da sensibilidade apresentada pelo método à variação das abstrações iniciais (b); e da velocidade de infiltração básica da água no solo (c). $\mathrm{i}_{\mathrm{i}}$ =intensidade instantânea de preci pitação; VI B =velocidade ou taxa de infiltração básica de água no solo; $I_{\mathrm{a}}=$ abstrações iniciais; ES = escoamento superficial; I $=$ infiltração acumulada; $\mathbf{t}_{1 \mathrm{a}}=$ duração das abstrações iniciais; $\mathbf{t}_{\mathbf{i n f}}=$ duração da infiltração; $\mathbf{t}=$ tempo para escoamento superficial máximo.

(a)

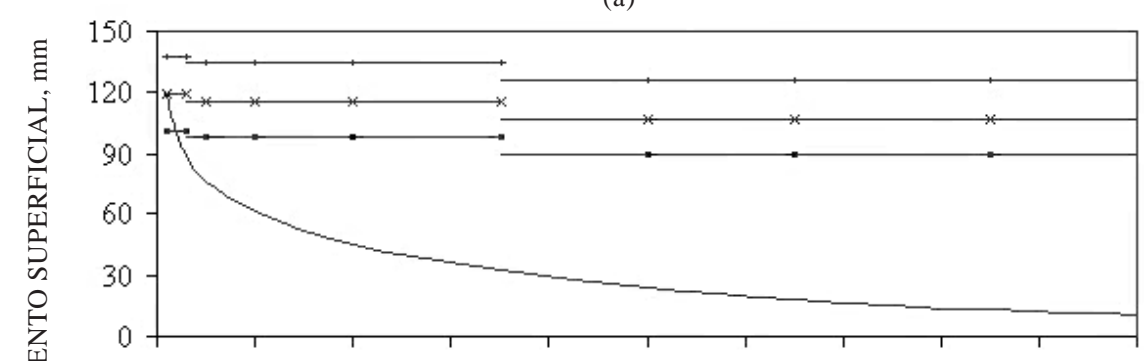

(b)

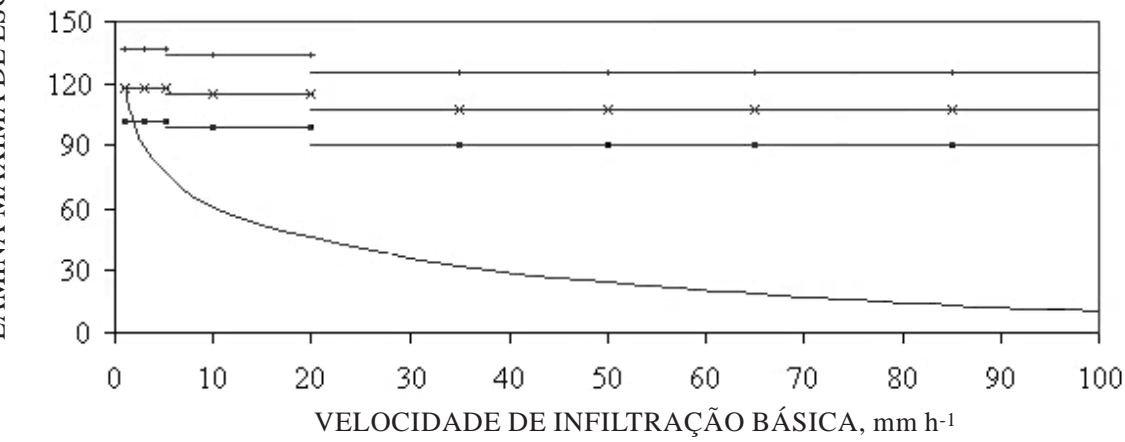

- Método Pruski et al (1997) $\rightarrow$ Método CN para 6 horas

Método CN para 12 horas - Método CN para 24 horas

Figura 3. Representação, para Belo Horizonte, da variação da lâmi na máxi ma de escoamento superficial com a VIB por meio dos métodos propostos por Pruski et al. (1997) e número da curva, utilizando os critérios apresentados por Pruski et al. (1997) (a) e SCS (b) para a classificação do solo nos grupos propostos pelo SCS-USDA para o método do número da curva. 
Os dois métodos estudados apresentaram redução nos valores delâmina máxima deescoamento superficial com o aumento da velocidade de infiltração básica da água no solo. No método $\mathrm{CN}$, porém, esta redução ocorreu de acordo com as faixas de VIB correspondentes aos grupos de sol os. A estratificação da lâmina máxima de escoamento superficial, segundo os quatro grupos de solos previstos pelo método, éuma das deficiências destee decorreda consideração da velocidade de infiltração da água no solo de forma indireta no modelo, ou seja, apenas para o seu enquadramento nos grupos previstos pel o método.

Estecomportamento não é evidenciado no método desenvolvido por Pruski et al. (1997), o qual semostrou mais sensível à variação da VIB , apresentando uma redução gradativa nos valores de lâmina máxima de escoamento superficial com o incremento dos valores da VIB, o que decorre do fato de o método calcular, para cada situação, o valor da chuva que provoca o máximo escoamento (equação 8). No método CN, entretanto, a precipitação à qual corresponde o máximo escoamento superficial constitui dado de entrada, devendo, portanto, a duração da preci pitação e a sua magnitude serem fornecidas pel o projetista.

O método CN apresentou, para uma mesma VIB, valores crescentes de escoamento superficial com o aumento da duração da chuva considerada, o que pode ser explicado pelo fato de precipitações mais longas, embora não tão intensas, apresentarem maior volume precipitado.

No quadro 2, observa-se que, para pequenos valores de VIB (até $2 \mathrm{~mm} \mathrm{~h}^{-1}$ ), os valores de escoamento superficial calculados pelos dois métodos apresentaram diferenças relativamente pequenas. Com o aumento da VIB, no entanto, ocorreu um aumento na diferença entre os valores da lâmina máxima de escoamento superficial nos dois métodos. Assim, para VIB igual a $1 \mathrm{~mm} \mathrm{~h}^{-1}$, ovalor da lâmina máxima de escoamento superficial obtido pelo método proposto por Pruski et al. (1997) (119,1 mm) aproximou-se do encontrado pel o método $\mathrm{CN}$ para a duração de $12 \mathrm{~h}(118,4 \mathrm{~mm})$, sendo superior ao encontrado para a duração de $6 \mathrm{~h}(101,2 \mathrm{~mm})$ e inferior ao obtido para a duração de $24 \mathrm{~h}(137,3 \mathrm{~mm})$. J á para a VIB de $2 \mathrm{~mm} \mathrm{~h}^{-1}$, oval or obtido pelo método proposto por Pruski et al. (1997) $(99,7 \mathrm{~mm}$ ) aproximou-se do obtido pelo método $\mathrm{CN}$ para a duração de $6 \mathrm{~h}(101,2 \mathrm{~mm})$ e foi inferior aos estimados para as durações de $12 \mathrm{~h}(118,4 \mathrm{~mm})$ e $24 \mathrm{~h}(137,3 \mathrm{~mm})$. Para VIB's iguais ou superiores a $2 \mathrm{~mm} \mathrm{~h}^{-1}$, o método proposto por Pruski et al. (1997) apresentou val ores inferiores aos do método CN para todas as durações de preci pitação estudadas. Para a cidade de U berlândia, o comportamento mostrou-se semel hante (Figura 4).

Para ambas as localidades, a diferença entre os val ores de escoamento superficial obtidos pel os dois métodos aumentou progressivamente com a duração da chuva e aumento na VIB. Com o método CN, obtiveram-se valores de lâmina máxima de escoamento superficial bastante elevados, mesmo para VIB's muito altas, e, para a local idade de Belo Horizonte, para a VIB de $210 \mathrm{~mm} \mathrm{~h}^{-1}$, os valores obtidos pelo método $\mathrm{CN}$ foram superiores aos encontrados pelo método proposto por Pruski et al. (1997) para a VIB de $10 \mathrm{~mm} \mathrm{~h}^{-1}$.

Considerando os valores da lâmina máxima de escoamento (Quadro 2) e os critérios propostos por Pruski et al. (1997) para a classificação do solo nos grupos estabelecidos pelo SCS-USDA, verifica-se que, para a VIB de $1 \mathrm{~mm} \mathrm{~h}^{-1}$, os val ores encontrados pelo método $\mathrm{CN}$ para as durações de precipitação de $6 \mathrm{~h}, 12 \mathrm{~h}$ e $24 \mathrm{~h}$ apresentaram, respectivamente, diferenças de-15,0; -0,6 e 15,3\% em relação àquel es obtidos pel o método proposto por Pruski et al. (1997). Para a VIB de $50 \mathrm{~mm} \mathrm{~h}^{-1}$, o método CN apresentou val ores superiores aos do Pruski et al. (1997) em 273, 344 e $421 \%$, respectivamente, para precipitações de 6,12 e 24 h de duração.

Utilizando o método proposto por Pruski et al. (1997), obtiveram-se valores de lâmina máxima de escoamento superficial muito baixos para al tas taxas de infiltração (Quadro 2 e Figuras 3 e 4). No quadro 2, evidencia-se uma lâmina máxima de escoamento superficial de $0,3 \mathrm{~mm}$ para a VIB de $210 \mathrm{~mm} \mathrm{~h}^{-1}$, o que corresponde ao comportamento observado sob condições reais, uma vez que, quando a intensidade de precipitação é igual ou inferior ao valor da velocidade de infiltração, não ocorre escoamento superficial. Pelo método $\mathrm{CN}$, esta redução não ocorreu deforma tão expressiva, sendo, mesmo para valores deVIB de $210 \mathrm{~mm} \mathrm{~h}^{-1}$, os val ores de lâmina máxima de escoamento superficial estimados foram superiores a $75 \mathrm{~mm} \mathrm{~h}^{-1}$.

O uso de diferentes critérios para a classificação do solo nos grupos propostos pelo SCS-USDA não promoveu alterações expressivas nos resultados obtidos. Pel o método de Pruski et al . (1997), ocorreu somente pequena variação no valor da lâmina máxima de escoamento superficial observada nas extremidades de cada faixa de VIB, enquanto, pelo método CN , observou-se apenas uma alteração na extensão das faixas, permanecendo os mesmos val ores delâmi na máxima deescoamento superficial cal culados. Estas pequenas variações deveram-se ao fato de que, no método CN, o valor da VIB não foi utilizado diretamente para obter o escoamento, mas apenas para estabelecer a faixa para utilização de cada um dos valores de $\mathrm{CN}$.

A quantificação precisa dos valores de escoamento superficial pode reduzir os custos das obras quenecessitam desses valores para seu correto dimensionamento, representando, portanto, um aumento na segurança da obra eeconomia decapital. Dessemodo, a aplicação de model os que representem melhor as condições impostas pela natureza é de extrema importância para as obras que dependam do conhecimento destas condições. 
(a)

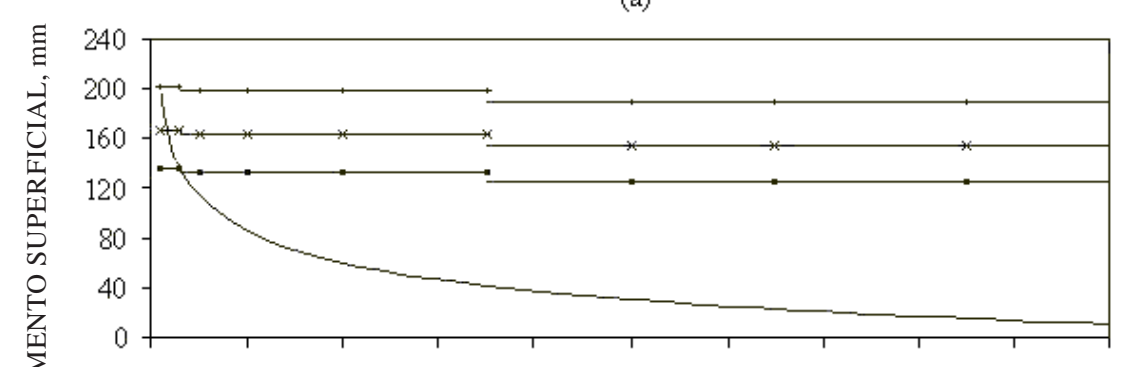

(b)

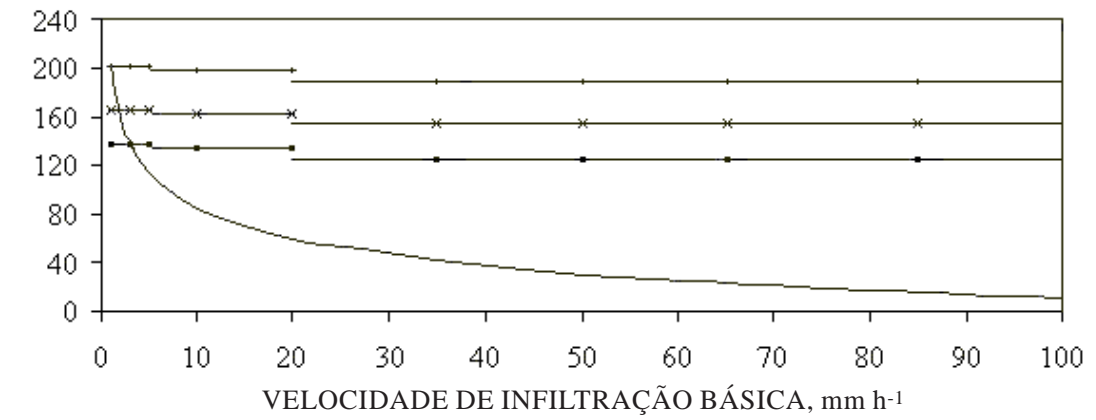

- Método Pruski et al. (1997) $\rightarrow$ Método CN para 6 horas

$\rightarrow$ Método CN para 12 horas $\rightarrow$ Método CN para 24 horas

Figura 4. Representação, para Uberlândia, da variação da lâmina máxima de escoamento superficial com a VIB por meio dos métodos propostos por Pruski et al. (1997) e número da curva, utilizando os critérios apresentados por Pruski et al. (1997) (a) e SCS (b) para a classificação do solo nos grupos propostos pelo SCS para o método do número da curva.

\section{CONCLUSÕES}

1. Os dois métodos para determinação do escoamento superficial apresentaram redução do escoamento superficial com o aumento na taxa de infiltração de água no sol o.

2. O método proposto por Pruski et al. (1997) descreveu o decaimento do escoamento superficial com o aumento da taxa de infiltração de forma mais condizente com a realidade.

3. O método proposto por Pruski et al. (1997) apresentou lâminas de escoamento superficial bastanteinferiores às do método do número da curva para el evadas taxas de infiltração de água no solo.

\section{LITERATURA CITADA}

BERTONI, J . \& LOMBARDI NETO, F. Conservação do solo. 3.ed. São Paulo, Í cone, 1990. 355p.

BONTA, J .V. \& RAO, A.R. Estimating peak flows from small agricultural watersheds. J . Irrig. Drain. Eng., 118:122-137, 1992.

HAWKINS, H.R. Infiltration and curve numbers, In: SYMPOSIUM ON WATERSHED MANAGEMENT, New York, 1980. Anais. New York, American Society of Civil Engineers, 1980. p.925-937.
LOMBARDI NETO, F.; BELLINAZZI J r., R. \& LEPSCH, I.F. Terraceamento agrícola. Campinas, Secretaria da Agricultura e do Abastecimento do Estado de São Paulo, Coordenadoria de Assistência Técnica I ntegral, 1994. 39p. (Boletim, 206)

MARTINS J r., D. Avaliação de um model o desimulação delâmina e vazão máxima de escoamento superficial. Viçosa, Universidade Federal de Viçosa, 1997. 86p. (Tese de Mestrado)

PINTO, F.A.; FERREIRA, P.A.; PRUSKI, F.F.; ALVES, A.R. \& CECON, P.R. Equações de chuvas intensas para algumas localidades do Estado de Minas Gerais. Eng. Agric., 16:91104, 1996.

PRUSKI, F.F.; FERREIRA, P.A.; RAMOS, M.M. \& CECON, P.R. A model to design level terraces. J . I rrig. Drain. Eng., 123:812, 1997.

RALLISON, R.E. Origin and evolution of the SCS Runoff equation, In: SYMPOSIUM ON WATERSHED MANAGEMENT, New York, 1980. Anais. New York Americam Society of Civil Engineers, 1980. p.912-924.

ROJ AS, R.M. Drenaje superficial de tierras agrícolas. Mérida, Venezuela, 1984. 96p. não publicada.

WELLS, L.G.; WARD, A.D.; MOORE, I.D. \& PHILLIPS, R.E. Comparison of four infiltration models in characterizing infiltration through surface mine profiles. Trans. Am. Soc. Agric. Eng., 29:785-793, 1986. 